CAHIERS DE

NARRATOLOGIE

\section{Cahiers de Narratologie}

Analyse et théorie narratives

25 | 2013

Humour et modernité dans les littératures de langues romanes du XIXe au XXIe siècle

\title{
L'humour d'Arturo Pérez Reverte face à une société en crise
}

\section{Carmen Aznar Pastor}

\section{(2) OpenEdition}

Journals

Édition électronique

URL : http://journals.openedition.org/narratologie/6786

DOI : 10.4000/narratologie.6786

ISSN : 1765-307X

Éditeur

LIRCES

Référence électronique

Carmen Aznar Pastor, "L'humour d'Arturo Pérez Reverte face à une société en crise », Cahiers de Narratologie [En ligne], 25 | 2013, mis en ligne le 20 décembre 2013, consulté le 19 avril 2019. URL http://journals.openedition.org/narratologie/6786; DOI : 10.4000/narratologie.6786

Ce document a été généré automatiquement le 19 avril 2019

Article L.111-1 du Code de la propriété intellectuelle. 


\title{
L'humour d'Arturo Pérez Reverte face à une société en crise
}

\author{
Carmen Aznar Pastor
}

1 Dans le cadre de ce séminaire, nous allons nous intéresser à l'un des écrivains espagnols contemporains le plus lu aussi bien en Espagne qu'à l'étranger. Auteur d'une vingtaine de romans (à mi-chemin entre polar et roman historique), publiés dans plus de cinquante pays et traduits en plus de 25 langues, Arturo Pérez Reverte a débuté sa vie professionnelle en tant que journaliste, travaillant pendant plus de vingt ans comme grand reporter. Même si, à partir de 1994, il abandonne le journalisme pour se consacrer exclusivement à la littérature, il n'a jamais oublié sa première vocation et a continué à publier, sans discontinuité depuis 1991, des chroniques hebdomadaires dans le magazine XLSemanal, distribué le dimanche comme supplément à 25 journaux espagnols et devenue une des sections les plus lues de la presse espagnole avec plus de quatre millions de lecteurs.

2 Ce sont précisément ces chroniques hebdomadaires qui font l'objet de notre étude et cela pour deux raisons. Tout d'abord, l'humour, sous différentes déclinaisons et modalités, y est omniprésent et devient ainsi un élément structurant et porteur de sens. Aussi, la notion de modernité et de progrès est un axe important dans la thématique abordée dans ces articles.

3 Dans un premier temps nous allons nous interroger sur la question générique et le problème des frontières en examinant le caractère littéraire des chroniques journalistiques de l'auteur. Puis nous analyserons les principales thématiques qui se dégagent de l'ensemble de ses articles tout en détaillant les procédés humoristiques que l'auteur manie avec virtuosité. Nous essayerons enfin de montrer quelle est l'éthique qui se dégage du parti pris esthétique adopté par l'auteur.

4 Nombreuses études ont été consacrées à la question du columnismo des écrivains espagnols en tant que genre littéraire ${ }^{1}$. Il a été défini comme un nouveau genre littéraire, un genre hybride dont les origines remontent au XIXe siècle espagnol avec les articles de Mariano José de Larra. Cette tradition d'écrivains journalistes se prolonge avec les 
écrivains de la génération de 98 et connaît un renouveau après 1975 avec le retour de la démocratie et de la liberté d'expression dans la presse espagnole.

Ce nouveau genre, dénommé articulismo, columnismo ou arti-cuento selon les périodes et les auteurs, s'est avéré être un format idéal pour l'expérimentation, un espace de liberté où s'entremêlent - en s'enrichissant mutuellement - journalisme et littérature. Aujourd'hui des écrivains reconnus comme Javier Marias, Juan José Millas, Antonio Gala, Antonio Muñoz Molina, Almuneda Grandes, Rosa Montero, Manuel Rivas ou encore Javier Cercas, publient régulièrement des chroniques dans la presse espagnole.

6 Par ailleurs, notre auteur, qui s'inscrit dans cette longue tradition, considère ses chroniques comme faisant partie intégrante de son œuvre littéraire ; ainsi, elles ont été recueillies en quatre volumes, dépassant de ce fait le caractère éphémère du texte de presse pour accéder au statut pérenne du livre. A.P. Reverte souligne également l'étroite relation qu'il y a entre ses chroniques et son œuvre romanesque ; en effet, la lecture des chroniques permet de mieux comprendre son univers romanesque et les chroniques sont une clé de lecture de ses romans.

7 Enfin, lorsque l'on considère l'ensemble de ses chroniques, on en perçoit très nettement le caractère fictionnel. On peut parler d'une fictionnalisation de l'auteur dans ses articles dans la mesure où Reverte fait du narrateur un personnage qui prend forme au fil des quelques 2000 pages du corpus. Le lecteur découvre ainsi un personnage qui prend de l'épaisseur et évolue au contact d'une réalité qu'il commente et dont il s'inspire ; il s'agit bel et bien d'un personnage de fiction, d'un héros qui incarne et défend une certaine vision du monde et nous fait penser à certains personnages de l'univers romanesque de Reverte. On entrevoit ainsi des correspondances entre ses chroniques et l'ensemble de ses romans.

8 C'est précisément ce personnage fictionnel qui donne leur unité (et leur sens) à l'ensemble des chroniques et qui nous servira de fil conducteur lors de notre étude.

Avant d'aborder notre analyse, arrêtons-nous quelques instants sur les titres des recueils pour tenter de déceler quelques éléments de réponse.

Le titre du premier recueil, Patente de corso - qui réunit les articles publiés entre 1993 et 1998 et peut se traduire comme « Lettre de course » - évoque l'idée d'avoir carte blanche, de bénéficier d'une autorisation pour faire ce qui est interdit aux autres. Le titre annonce une certaine liberté de ton et l'on voit se dessiner la figure d'un personnage - le pirate qui vit hors la loi mais qui incarne en même temps l'archétype du héros romantique, un hors la loi qui dérange mais fascine à la fois.

11 Con ánimo de ofender, titre du deuxième volume qui rassemble les articles édités entre 1998 et 2001, prend le contre-pied de l'expression "sans vouloir offenser " et souligne la volonté de provoquer, de choquer pour faire réagir et préfigure un ton impertinent voire insolent.

Dans le troisième volume - ensemble de chroniques publiées entre 2001 et 2005 - intitulé No me cogeréis vivo (Vous ne m'attraperez pas vivant) émerge l'idée de courage et la volonté d'aller jusqu'au bout malgré le risque et le danger.

Cuando éramos honrados mercenarios (Lorsque nous étions des honnêtes mercenaires), dernier recueil incluant les articles publiés entre 2005 et 2009, fait renaître les combattants de métier, les soldat à la solde et cependant fidèles à certains valeurs, dont l'honnêteté. Une pointe de nostalgie transparait aussi, la nostalgie d'une époque où l'on respectait encore un code d'honneur, une époque où la droiture morale avait encore un sens. 
situations absurdes qu'il n'hésite pas à tourner en dérision. Dans le but d'en finir avec les mauvais traitements endurés par les immigrés clandestins qui essayent de traverser nos frontières, il suggère de créer un nouveau corps dans la gendarmerie - une sorte d'ONG qu'il baptise Gendarmes sans Frontières - accomplissant sa mission fermement mais avec amour et compassion. Ces gendarmes sans frontières devraient adresser aux immigrés, en français, anglais, arabe et swahili, et sur un ton mesuré, la phrase qui suit: Je vous prie monsieur de ne pas transgresser la frontière et de retourner chez vous, si vous le voulez bien, s'il vous plaît, merci. Si malgré cela l'immigré persiste, il faudrait passer au plan B :

Les attraper par les épaules sans violence mais avec fermeté démocratique, les embrasser sur la bouche vigoureusement, et ensuite leur indiquer le chemin vers les bus qui les conduiront aux centres d'accueil ; ou bien, pour éviter une perte de temps, les accompagner directement prendre l'avion ou le bateau vers la péninsule. Le tout, bien entendu, en présence d'une commission des droits de l'homme du Congrès ${ }^{4}$.

Dans sa chronique Mourir comme un porc il se moque de ceux qui dénoncent la barbarie de la traditionnelle fête espagnole où l'on tue le cochon. Il préconise le protocole qui suit afin d'adoucir la mort de l'animal : 
Le cochon doit être drogué et électrocuté partiellement afin d'atténuer sa souffrance; lors de son trajet vers la scène du calvaire, on devrait lui mettre des lunettes de soleil pour éviter que la lumière du jour ne blesse sa délicate rétine. Une fois dans le lieu du crime, on lui offrira un petit pétard, un petit wisky un valium et si possible une truffe. Il sera ensuite égorgé avec toute la délicatesse du monde. Après ça, tous les cochons européens voudront mourir en Espagne et nous exporterons du lard écologique (Bio-bacon) et nous mangerons des boudins la conscience tranquille ${ }^{5}$. spécialité, au même titre que la paella et la corrida :

Si l'Espagne exportait des idiots - elle le fait parfois mais sans organisation ni méthode - nous serions la première puissance mondiale. L'idiot espagnol est un idiot réputé et de première qualité. Si l'idiot espagnol disparaissait en tant qu'espèce, ce serait aussi désastreux que la disparition du taureau de combat (...). Une partie importante de notre identité culturelle partirait en vrille ${ }^{6}$.

sottise est en fait un symptôme d'un phénomène bien plus grave et inquiétant: la fadaise, la futilité et la vacuité inhérente à la société postmoderne; autrement dit, l'absence de grandeur qui résulte de l'étiolement de valeurs et de la perte de discernement. Ainsi, dans la chronique intitulée Toasts à la crème il dépeint la superficialité qui envahit tous les sphères de notre société de consommation et de l'apparence :

Nous voulons du lait pasteurisé, écrémé et aseptisé. Nous préférons les fruits bien gros, brillants et enrobés de cire, les biftecks sans graisse et des trucs de la sorte. Avec les gens, il arrive la même chose. Le monde est rempli d'individus brillants comme des pommes, maigres comme ces biftecks en plastique qui n'ont aucun goût. On regarde autour de soi, on goûte un peu tout. Et l'on finit par constater que presque tout, les pommes, les biftecks, les gens, a la même saveur, une saveur indéfinissable et quelconque. C'est peut-être ça le progrès. Une appétissante pastèque à la peau luisante et à la chair rouge mais aussi savoureuse qu'un concombre?

Si dans le domaine sociétal l'auteur se contente de railleries, de dérision, et le ton reste relativement bienveillant, il n'en va pas de même lorsqu'il aborde le monde politique. Devant la corruption et l'incompétence des hommes politiques, face à la bêtise des nationalistes qui sont en train de diviser l'Espagne, le langage se fait plus mordant et sarcastique.

Les excès des nationalismes périphériques lui inspirent des pages d'une ironie féroce qui cache mal l'indignation face à la gravité des conséquences pour l'Espagne :

Il est bien plus utile, et surtout bien plus fun, que chaque espagnol apprenne exclusivement l'histoire, la langue, la littérature de sa petite ville, de son petit village, de son petit quartier et qu'il lise Le Quijote uniquement traduit dans sa langue régionale. A l'aube du XXIe siècle tout cela est parfaitement normal, c'est faire preuve d'un respect exquis face à la multi pluralité plurielle du pluralisme plurinational plurilingue et plurimorphe de notre pays si pluriel qu'il fait l'admiration de l'Europe et du monde entier; ils se demandent comment c'est possible qu'ils n'aient pas eu avant nous cette idée géniale qui consiste à anéantir une entité historique en l'espace de six mois 8 .

La conséquence de cette dislocation est la transformation de l'Espagne en « une fédération de petits royaumes, une soi-disant monarchie plurinationale ou une maison close où le moins dégourdi sort avec la plus moche ${ }^{9}$ ». 
Mais ce sont les hommes politiques qui suscitent les réactions les plus vives chez l'auteur. Dans son article Pieds de ministre - jeu de mots à partir de l'expression " pieds de cochon ", le remplacement de « cochon " par « ministre » donne le ton de l'article - il tire à boulets rouges sur la classe politique espagnole; employant un registre de langue parlé et familier, il classe les politiciens espagnols en trois catégories: "les charlatans, les baratineurs et les fils de pute ${ }^{10} »$.

Lorsqu'il s'agit de critiquer l'immoralité et l'incompétence de la classe politique espagnole en général, le ton se fait de plus en plus virulent:

Permettez-moi de vous tutoyer, bande d'imbéciles, bande de voyous et de voleurs, tous autant que vous êtes : intégristes analphabètes de droite, démagogues illettrés de gauche. Vous qui avez fait de cet auto-complaisant pays de merde, un pays encore plus merdique ${ }^{11}$.

L'auteur consacre d'ailleurs plusieurs articles à justifier son recours à l'insulte qu'il définit comme :

un soulagement, un acte de violence verbale où l'on vide l'estomac et le cœur, un mot qui frappe comme un coup de fouet pour cracher tout le dégoût et la colère accumulés au cours de siècles de massacres et de haines fratricides, d'impuissance, d'oppression, d'ignorance, de jalousie, d'orgueil et de barbarie. C'est une déclaration de principes pour régler ses comptes avec le monde entier ${ }^{12}$.

Il faut chercher les raisons de la déchéance espagnole dans son passé ; c'est là que se trouvent les carences historiques - le manque de patriotisme, le mépris de la tradition, l'ignorance, l'incurie - qui ont favorisé la violence et la barbarie et ont fait de l'Espagne ce qu'elle est aujourd'hui.

31 Lorsqu'il décrit la réalité espagnole comme profondément dégradée, Reverte reste profondément espagnol ; à travers cette vision lucide et désespérée d'une Espagne en déclin, prisonnière de sa stupidité, son inculture et sa cruauté, il s'inscrit dans la lignée directe de Quevedo, Larra, Joaquin Costa, Unamuno ou Machado. Il reprend à son compte le débat intellectuel concernant l'identité espagnole - le Problème espagnol - et sa problématique - y a-t-il une essence espagnole?

Il actualise en même temps l'image des deux Espagnes qui s'affrontent dans une lutte fratricide. Dans la chronique Le gourdin et le canif l'auteur s'inspire du tableau de Goya Duel à coup de gourdins qu'il interprète comme le symbole de la lutte fratricide entre espagnols :

Ce n'est pas vrai que la quintessence de notre race est en train de disparaître. La société de consommation, le vernis de civilisation, le soi-disant européisme, l'antenne parabolique et la versicolore Iberia peuvent nous induire en erreur. Nous inspirer une fausse impression de modernité, de changement de notre essence si authentique, si orthodoxe, si virile et si sympathique. La réalité est que, dans ce pays béni des Dieux, nous sommes toujours aussi doués pour nous taper dessous, à la moindre occasion, et nous le faisons avec un acharnement consciencieux et ancestral, une cruauté bien à nous. Ce satané sourd (Goya) a peint notre âme à la perfection. Au fond, tout cela est très drôle, même si l'on rit jaune ${ }^{13}$.

L'on retrouve ici l'idée d'une Espagne qui n'a jamais rejoint une modernité incarnée par Prométhée - c'est-à-dire, le triomphe de la raison et la recherche du bien commun - et qui a adopté un postmodernisme incarné par Narcisse - autrement dit, la perte de la vision collective et l'individualisme.

Face à la perte des valeurs et à la dislocation de cette postmodernité, le narrateur propose un retour aux valeurs qui lui sont chères : l'honnêteté et l'honneur qu'il définit comme des bastions nécessaires pour se protéger de la médiocrité, la stupidité ou le désespoir. 
défendre sa propre éthique. Les qualités morales qui définissent ce personnage sont le courage, l'honnêteté, la franchise, la loyauté, la conséquence; il est en quelque sorte la réplique contre-spéculaire et antinomique de la réalité. Il se présente comme un héros qui, tel un chevalier de la Renaissance, manie l'épée et la plume; ou plus exactement un soldat qui manie la plume comme une arme. Un personnage paré de toutes les vertus et qui, du haut de sa supériorité morale, dénonce la décadence d'une société en crise. Il est par moments un homme arrogant, agaçant, incisif, mordant; un homme en colère et moralisateur qui défend des valeurs étiques supérieures, habituelles dans le passé mais caduques aujourd'hui. Mais c'est aussi un héros «humain» qui, avec les années, se montre fatigué et désenchanté. A l'image du capitaine Alatriste, le héros est un soldat désabusé mais fidèle à ses principes. 
éfinitive, il s'agit du même postulat éthique que Arturo Pérez Reverte développe dans son univers romanesque. En cela nous pouvons affirmer qu'il existe une continuité et une cohérence entre l'œuvre romanesque et les chroniques journalistiques. Cela prouve aussi qu'il n'y a pas de frontière étanche entre les deux pratiques d'écriture et confirme le caractère littéraire des articles.

\section{NOTES}

1. Voir l'œuvre collective devenue une référence en la matière, Grohmann, Alexis y Steenmeijer, Maarten, El Columnismo de escritores españoles (1975-2005), Madrid, Verbum, 2006, 197 pp.

2. Arturo Pérez Reverte, «El síndrome Viracocha» in Patente de corso, Madrid, Santillana Ediciones Generales, S.L, 2010, p. 84.

3. Arturo Pérez Reverte, «Sin moneda para Caronte " in Patente de corso, Madrid, Santillana Ediciones Generales, S.L, 2010, p. 55.

4. Arturo Pérez Reverte, "Picoletos sin Fronteras " in Cuando éramos honrados mercenarios, Madrid, Santillana Ediciones Generales, S.L, 2011, p. 34.

5. Arturo Pérez Reverte, "Morir como un cerdo» in Patente de corso, Madrid, Santillana Ediciones Generales, S.L, 2010, p. 310.

6. Arturo Pérez Reverte, "Tontos (y tontas) de pata negra " in Cuando éramos honrados mercenarios, Madrid, Santillana Ediciones Generales, S.L, 2011, p. 606.

7. Arturo Pérez Reverte, «Tostadas de nata» in Patente de corso, Madrid, Santillana Ediciones Generales, S.L, 2010, p. 429.

8. Arturo Pérez Reverte, «Indíbil y Mandoni» in Patente de corso, Madrid, Santillana Ediciones Generales, S.L, 2010, p. 465.

9. Ibidem, p. 466.

10. Arturo Pérez Reverte, "Manitas de ministro» in Cuando éramos honrados mercenarios, Madrid, Santillana Ediciones Generales, S.L, 2011, p. 45.

11. Arturo Pérez Reverte, «Permitidme tutearos, imbéciles» in Cuando éramos honrados mercenarios, Madrid, Santillana Ediciones Generales, S.L, 2011, p. 367.

12. Arturo Pérez Reverte, «El insulto» in Patente de corso, Madrid, Santillana Ediciones Generales, S.L, 2010, p. 126.

13. Arturo Pérez Reverte, «El garrote y la navaje» in Patente de corso, Madrid, Santillana Ediciones Generales, S.L, 2010, p. 41.

\section{RÉSUMÉS}

Arturo Pérez Reverte brosse dans ses chroniques un portrait sans concession de la société espagnole contemporaine. Tout en mettant en évidence les failles d'un pays en crise, qui est passé de l'euphorie à l'effondrement en quelques années, il critique la décadence d'une 
modernité en crise. Et il le fait avec une prose mordante ; s'inspirant des héros de ses romans, il s'invente un personnage audacieux, arrogant, impertinent qui cherche à provoquer et à éveiller les consciences. Pour cela, il explore tous les registres de l'humour (l'ironie, le sarcasme, la parodie, l'humour noir) et n'hésite pas à aller jusqu'à l'insolence. Son humour, très personnel et actuel, s'inscrit en même temps dans la tradition littéraire espagnole.

Arturo Pérez Reverte esboza en sus columnas un retrato sin concesiones de la sociedad española contemporánea. Al mismo tiempo que pone en evidencia los fallos de un país en crisis, que ha pasado de la euforia al desmoronamiento en pocos años, critica la decadencia de una modernidad en crisis. Y lo hace con una prosa mordaz; inspirándose en los héroes de sus novelas, se inventa un personaje audaz, arrogante, impertinente que busca la provocación y el despertar de las conciencias. Para ello explora todos los registros del humor (la ironía, el sarcasmo, la parodia, el humor negro) y no duda en ir hasta la insolencia. Su humor, muy personal y actual, se inscribe al mismo tiempo en la tradición literaria española.

\section{INDEX}

Index chronologique : XXe siècle, XXIe siècle

Index géographique : Espagne

Mots-clés : littérature espagnole, columnismo, humour, Arturo Pérez Reverte

\section{AUTEUR}

\section{CARMEN AZNAR PASTOR}

Professeur agrégé d'espagnol en Classes Préparatoires aux Grandes Écoles au Lycée International de Valbonne ; chargée de cours à l'Université Nice Sophia Antipolis ; thèse de Doctorat sur «L'écriture du voyage chez Camilo José Cela »; membre du LIRCES. Articles du même auteur : -Récit et éthique dans l'écriture du voyage de Camilo José Cela,Cahiers de Narratologie, $\mathrm{N}^{\circ} 12$, mis en ligne le 20 avril 2005

-Los libros de viaje de Camilo José Cela, Revue Quimera, N 246-247, Août 2004, Barcelona -Los umbrales del relato : cuatro calas en el tiempo in Relatos de viajes contemporáneos por España y Portugal, Madrid, Verbum, 2004

-Le paratexte dans les récits de C.J.Cela, Revue IRIS ; 1998, Université Montpellier III, 1998. Communication du même auteur :

Les rapports entre la voix narrative et le point de vue, Cahiers de Narratologie ${ }^{\circ} 10$; CNA, Université de Nice, 2001 